\title{
La condición de vulnerabilidad de los migrantes en tránsito por la ruta del Occidente de México. Una propuesta de categorización
}

\author{
Olga Aikin Araluce* \\ Adriana González Arias
}

Recepción: 15 de junio de 2017 Aceptación: 4 de septiembre de 2017

Resumen El presente artículo hace una breve revisión de la migración de tránsito por la ruta del Occidente de México -sus características, sus actores y su perfil, el contexto, las causas migratorias, los riesgos- para analizar las condiciones de vulnerabilidad de los migrantes que por ella transitan. Con base en lo anterior, se hace una propuesta conceptual de vulnerabilidad, como concepto teórico operacionalizable, y se elaboran múltiples categorías para dicho término. Esta investigación -basada en un trabajo de campo más amplio- se realiza con el fin de identificar la red de condiciones y actores que vulneran a los migrantes en diferentes ámbitos durante su tránsito por la ruta Occidente, que ha sido poco estudiada a pesar de su importancia en la migración de tránsito por México.

PALABRAS CLAVE: migración de tránsito, ruta Occidente, vulnerabilidad, condiciones migratorias.

Abstract The present article makes a brief review of the phenomenon of transit migration through a route located in Western Mexico -as well as its characteristics, its actors and its profiles, the context, the migratory causes and the risks it has- in order to analyze the conditions of vulnerability of the migrants that go through it. Based on the aforementioned studies, a conceptual proposal of vulnerability as a theoretically operable concept is made, and multiple categories are elaborated for this particular term. This research -based on a broader field work- was carried out with the purpose of identifying the network of conditions and actors that make migrants vulnerable in different areas during their transit through the West route, which has not been studied enough despite its importance in the transit migration in Mexico.

KEY WORDS: transit migration, Western route, vulnerability, migration conditions.

Las autoras son profesoras-investigadoras del Instituto Tecnológico y de Estudios Superiores de Occidente (ITESo), adri@iteso.mx, oaikin@iteso.mx 


\section{Introducción}

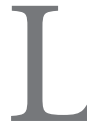

a visibilización de la migración de tránsito por México, principalmente de centroamericanos, se da a partir de la década de los noventa del siglo pasado. Sin embargo, la literatura académica menciona este fenómeno desde los ochenta, cuando estos flujos arribaban a México en busca de refugio o con la intención de llegar a Estados Unidos por causa de las guerras civiles de la época. A partir del año 2000, dicho fenómeno ha sido objeto de denuncia nacional e internacional debido a las múltiples violaciones de derechos humanos de las que estas personas han sido víctimas.

En el estudio de la migración de tránsito por México se ha observado que en las rutas que siguen los migrantes centroamericanos han confluido flujos de mexicanos que también tienen el objetivo de llegar a Estados Unidos y viven experiencias similares de vulnerabilidad por las condiciones en que realizan estos trayectos en su experiencia migratoria.

La literatura académica analiza, desde la óptica de la sociología, la antropología, la economía o la ciencia política, las rutas que se transitan, el perfil del migrante en tránsito y sus estrategias de supervivencia durante el camino; las redes sociales en que se apoya, las redes criminales que lo acechan, sus grupos de apoyo, la discriminación o el maltrato, las condiciones de violencia estructural de las que huyen, las violaciones a sus derechos humanos y la política migratoria que, a nivel nacional o regional, adoptan los gobiernos involucrados como forma de regular el fenómeno.

A partir de la profundización del fenómeno migratorio de tránsito por México y la elaboración de un estado del arte de la literatura que lo estudia, se observa qué poco sabemos acerca de una de las rutas cada vez más utilizada por los migrantes: la ruta del Occidente de México, cuyo trazo presentamos en la figura $1 .{ }^{1}$ Por lo tanto, nos surgió la inquietud de hacer una investigación específica sobre dicha ruta a partir de la descripción y el análisis de las condiciones de movilidad de los grupos migratorios que la transitan camino a la frontera norte. Para ello describimos la ruta y analizamos los actores que por ella viajan, con especial énfasis en los riesgos a la seguridad del migrante y en sus capacidades para afrontarlos. A partir de los conceptos de riesgo y capacidades o recursos, logramos construir varias categorías de vulnerabilidad que nos ayudan a comprender las condiciones del viaje para los distintos grupos migratorios.

En este artículo analizamos las condiciones de vulnerabilidad de los migrantes que transitan la mencionada ruta. En los primeros dos apartados presentamos el contexto general del viaje, las causas que impulsan a esta población a emigrar, así

La ruta de Occidente inicia en Irapuato, Guanajuato, atraviesa los estados de Jalisco, Nayarit y Sinaloa y acaba en Sonora o Baja California, siguiendo el trazado ferroviario. 


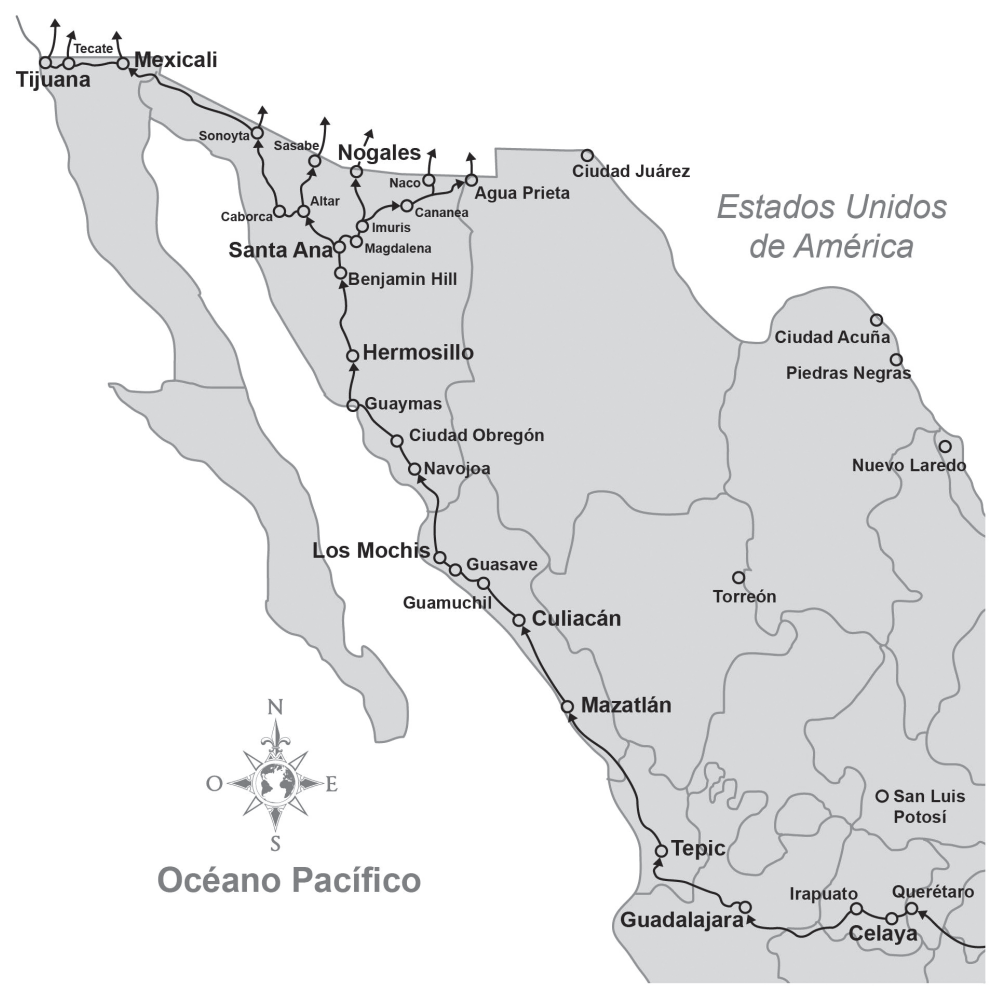

Fuente: Elaboración propia a partir de información obtenida en la investigación de campo.

como los peligros y riesgos que enfrentan a lo largo de la ruta occidental. En los últimos dos apartados presentamos nuestra propuesta conceptual de vulnerabilidad, como concepto teórico operacionalizable, y varias categorías de vulnerabilidad. Finalmente, a modo de conclusión, presentamos unas consideraciones finales y los agradecimientos a las personas e instituciones que han apoyado esta investigación.

Todo lo que presentamos en este artículo lo hemos trabajado a partir de una investigación más amplia llamada "Migración de tránsito por la ruta del occidente de México: actores, riesgos y perfiles de vulnerabilidad", la cual hemos presentado en diversas ponencias y publicaciones como parte de los resultados obtenidos de principios del año 2014 a finales de 2015. 


\section{Contextos y causas migratorias}

En la investigación ${ }^{2}$ de campo encontramos cuatro grandes grupos que transitan la ruta hacia el norte: mexicanos, hondureños, salvadoreños y guatemaltecos. Buscamos hacer un conjunto de entrevistas mínimamente representativas de todas las nacionalidades, si bien el grupo más numeroso encontrado fue el de los hondureños $(41.7 \%)$, seguido por guatemaltecos $(21.5 \%)$, salvadoreños $(20.2 \%)$ y mexicanos (16.4\%). Estos porcentajes no reflejan la composición cuantitativa del flujo porque no era nuestro objetivo y ya se ha presentado esa información en otros estudios, como la Encuesta sobre Migración en la Frontera Sur 2012 y el reporte de FM4 Paso Libre de 2013.

El perfil predominante entre los migrantes entrevistados fue el del hombre adulto de entre 20 y 25 años de edad (80.7\% respondía a estas características). En contraste, los grupos minoritarios encontrados fueron los de mujeres migrantes (solo $19.23 \%$ del total), menores de edad ( $8 \%$ del total) y mayores de 50 años (solo 5\%).

$\mathrm{Al}$ revisar las causas de la migración observamos que una parte importante de los entrevistados respondían al perfil del migrante por razones económicas (42.3\%) y $9 \%$ manifestó haber salido de su lugar de origen por cuestiones de violencia, principalmente la agresión propia de un ambiente social permeado por el narcotráfico y el pandillerismo. No obstante, para el $30.8 \%$ de ellos la mezcla de ambos motivos -económicos y de violencia- fue lo que determinó la emigración. En este sentido, es importante señalar que la pobreza es el motor principal de la expulsión de esta población, variable que a menudo se conjunta y refuerza con el factor violencia.

CUADRO 1. Causas de la migración por nacionalidad

\begin{tabular}{|c|c|c|c|c|c|c|c|c|c|}
\hline \multirow[t]{2}{*}{$\begin{array}{c}\text { Causas } \\
\text { de migración }\end{array}$} & \multicolumn{2}{|c|}{ Honduras } & \multicolumn{2}{|c|}{ Guatemala } & \multicolumn{2}{|c|}{ El Salvador } & \multicolumn{2}{|c|}{ México } & \multirow{2}{*}{$\begin{array}{c}\text { Total } \\
\text { Porcentaje }\end{array}$} \\
\hline & Frecuencia & Porcentaje & Frecuencia & Porcentaje & Frecuencia & Porcentaje & Frecuencia & Porcentaje & \\
\hline Violencia & 4 & 13 & 0 & 0 & 3 & 19 & 0 & 0 & 9 \\
\hline $\begin{array}{l}\text { Pobreza y falta } \\
\text { de trabajo }\end{array}$ & 11 & 34 & 10 & 59 & 6 & 37 & 6 & 46.1 & 42.2 \\
\hline $\begin{array}{l}\text { Violencia/ } \\
\text { pobreza y falta } \\
\text { de trabajo }\end{array}$ & 12 & 37 & 3 & 18 & 7 & 44 & 2 & 15.4 & 30.8 \\
\hline $\begin{array}{l}\text { Otros motivos } \\
\text { personales }\end{array}$ & 5 & 16 & 4 & 23 & 0 & 0 & 5 & 38.5 & 18 \\
\hline Total & 32 & 100 & 17 & 100 & 16 & 100 & 13 & 100 & 100 \\
\hline
\end{tabular}

Fuente: Elaboración propia a partir de datos del trabajo de campo.

La investigación, básicamente de corte cualitativo, se basó en un trabajo de campo realizado entre enero y mayo de 2014. En él aplicamos 78 entrevistas semiestructuradas a migrantes de tránsito hacia la frontera norte -46 en Guadalajara, Jalisco, y 32 en Nogales, Sonora- e hicimos cinco entrevistas a informantes clave -investigadores y encargados de comedores para migrantes. 
Hacer una breve referencia al problema de la violencia en Centroamérica resulta importante por ser esta una causa decisiva en la expulsión migratoria. La violencia en esta región data de varias décadas atrás, ya que estos países nunca se recuperaron de las guerras civiles que acabaron en los años noventa. No obstante, más recientemente, y en especial a partir de los años 2000 y 2006, la ola de violencia se exacerbó y países como Belice, El Salvador, Guatemala y Honduras experimentan actualmente los mayores niveles de homicidios en el mundo (UNDOC, 2012).

En Honduras, el país más afectado, la tasa nacional de homicidios en 2011 (92 por cada 100,000 habitantes) ha tenido el registro más alto de los últimos años (UNDOC, 2012). La hipótesis que propone para explicarlo la Oficina de las Naciones Unidas contra la Droga y el Delito (UNDOC) es que el flujo de cocaína por la región aumentó exponencialmente a partir del año 2000, y nuevamente en 2006 debido a la política del presidente Calderón de lucha contra el narcotráfico en territorio mexicano, que desvió gran parte del flujo de drogas a la zona centroamericana; esto creó nuevas rutas que atravesaban áreas controladas por los grupos de delincuencia organizada locales, lo que alteró el equilibrio de poder entre ellos y elevó el nivel de violencia. El flujo de grandes volúmenes de cocaína aumentó la competencia por el control territorial. Además de los grupos de transportistas de cocaína, existen en esta zona, y en México, grupos con arraigo territorial que gravan toda la actividad criminal en sus territorios y colaboran o rivalizan con las grandes redes transnacionales de transportistas de droga; entre ellos se disputan los mercados. A la violencia que generan estas luchas entre grupos se añade la presencia de pandillas conocidas como las maras que, si bien no tienen conexión con el tráfico de cocaína, realizan actividades masivas de extorsión y luchas de poder por el control local.

Así, la violencia en Centroamérica es generada principalmente por grupos delictivos de distinta escala y naturaleza que trafican con drogas, migrantes y armas y que también han incursionado en la trata de personas (UNDOC, 2012). Todo ello genera elevados niveles de violencia y hace a la población civil altamente vulnerable a este tipo de problemáticas. En esto tienen una causa más por la cual emigrar en busca de una vida mejor en otro país.

\section{Riesgos y peligros en la ruta del Occidente mexicano}

Los riesgos de las rutas migratorias pueden ser de dos tipos en general: circunstanciales y arbitrarios. Los primeros se derivan de los peligros propios del transporte utilizado y de las características físicas de las rutas; en concreto, hechos como atravesar zonas despobladas donde pueden encontrar animales venenosos, extensiones de agua peligrosas para quienes no sepan nadar o la carencia de calzado o ropa adecuada para soportar las inclemencias del terreno y el clima. Asimismo, los accidentes 
de carretera son frecuentes, así como las caídas de trenes o camiones de carga en movimiento. Por otro lado, los riesgos arbitrarios se refieren a todas las agresiones contra la vida e integridad de la persona migrante, tanto físicas como psicológicas, robos, extorsiones, secuestros e incluso trata de personas. Son perpetradas en su mayoría por el crimen organizado y autoridades (González, 2012).

Según Foote y Small (2013), en la ruta de Occidente el crimen organizado está menos presente, pero la extorsión por parte de la policía es muy elevada y las condiciones climáticas de la ruta son más peligrosas que en otros itinerarios. Esta afirmación coincide con nuestro hallazgo de que los problemas más recurrentes reportados por los migrantes fueron los abusos cometidos por autoridades - policías federales y locales, pero también agentes migratorios que extorsionan a los migrantes- y los problemas de salud derivados de dichos abusos y de las condiciones áridas de la ruta.

CUADRO 2. Problemas y abusos experimentados por los migrantes en la ruta Occidente

Tipos de problemas/abusos sufridos durante el viaje

Extorsión por policías y agentes de migración

Agresión de grupos criminales

(pandilleros, mafias y narcotraficantes)

Agresión de ciudadanos mexicanos

Accidentes en el tren o por el camino

Problemas de salud
Porcentaje de entrevistados que los sufrieron
37

32

20.5

69

Fuente: Elaboración propia con datos del trabajo de campo de esta investigación.

En las entrevistas, el 37\% de los entrevistados afirmó que fue agredido por el crimen organizado, mafias y grupos pandilleros durante el tránsito por México, si bien casi $73 \%$ de las agresiones ocurrieron en la parte sur y centro del país. El 10\% de los migrantes entrevistados señala como zonas "calientes" Sinaloa (específicamente Mazatlán, por la presencia de pandillas) y Sonora (específicamente Altar, Nogales y otros puntos de la frontera debido a la presencia de crimen organizado y mafias). Estos datos no nos permiten afirmar en forma contundente que la ruta del Occidente sea la más segura, o cuantificar puntualmente la incidencia de la agresión por parte de los distintos grupos criminales. No obstante, la mayoría de los migrantes afirma que optaron por esta ruta por presentar condiciones más seguras: concretamente, menos presencia del crimen organizado y de operativos migratorios. Si bien esta parece ser la percepción que predomina entre ellos, las condiciones de la ruta podrían agravarse en el futuro si tenemos en cuenta que, en la medida en que los migrantes ajustan los itinerarios del viaje en búsqueda de mayor seguridad, 
sus victimarios también lo hacen, por lo que el abuso tiende a perseguirlos (Casillas, 2008; Foote \& Small, 2013).

Si bien las extorsiones de autoridades y la agresión por parte de grupos criminales son la fuente de mayor preocupación de los migrantes, el 32\% de ellos afirma haber sido agredido por ciudadanos mexicanos mediante insultos, robos, extorsiones y en otras formas. Los eventos más frecuentes se produjeron con trabajadores de la línea ferroviaria, más que en encuentros casuales con ciudadanos mexicanos.

Los accidentes de tren o durante el camino representan un cuarto grupo de problemas que enfrentan los migrantes en su tránsito por este corredor, $20.5 \%$ manifiesta haberlos sufrido. Asimismo, la mayoría (69\%) presentó problemas de salud durante el viaje, condición que podríamos explicar como consecuencia, en gran medida, de los riesgos circunstanciales.

La frontera occidental norte presenta actualmente un nivel de riesgo y peligrosidad que antes no tenía. Ello se debe a que ha habido una modificación en las rutas de acceso clandestino a Estados Unidos. Si a mediados de la década de los noventa del siglo pasado $45 \%$ de los flujos migratorios pasaban por Tijuana y $15 \%$ por Ciudad Juárez, la tendencia ha cambiado enormemente en los últimos veinte años dado el control migratorio ejercido por Estados Unidos en su frontera (EMIF Norte, 2011). Operaciones como Hold the Line (1993 en la zona El Paso-Ciudad Juárez), Gatekeeper (1994, para la zona San Diego-Tijuana), Safeguard (para Arizona-Nogales) o la Arizona Border Control Initiative de 2004 han empujado a los flujos migratorios clandestinos de las urbes fronterizas más grandes a zonas desérticas e inhóspitas con climas muy secos y temperaturas extremas, potencialmente letales para cualquier migrante que aspire a cruzar. Puntos del estado de Sonora como San Luis Río Colorado, Altar, Agua Prieta, El Sásabe, Sonoyta, Sáric, Naco y Cananea han cobrado auge migratorio.

El aumento de flujos por zonas desérticas, el auge de redes de tráfico de migrantes, así como la presencia de distintos grupos delictivos, muchos de los cuales también lucran con los migrantes, hacen de esta frontera occidental un lugar muy peligroso para estos.

Si bien en esta ruta no se ha mencionado la existencia de secuestros masivos como en el sur de México, ocurren incidentes que se podrían equiparar con secuestros comunes. "A menudo los coyotes confinan a los migrantes en casas, les quitan sus medios de comunicación y los tienen a su disposición el tiempo que quieran. Esto quizás sí puede ser un secuestro, aunque con más consentimiento en principio" (Machuca, 2014). Castillo (2003) menciona que algunos migrantes tienen que recurrir a apoyos indirectos para organizar su desplazamiento, como son "intermediarios, conductores, prestadores de servicios diversos e incluso bandas organizadas que participan en el desplazamiento de migrantes indocumentados" (Castillo, 2003 , pp. 20-21). Estos apoyos pueden beneficiar a los migrantes, pero a menudo 
durante los procesos de tránsito son víctimas de abusos verbales, físicos y sexuales, así como de robo, secuestro o alojamiento forzado por parte de bandas organizadas. De esta manera, las prácticas lucrativas que caracterizan el contrabando o tráfico de migrantes se convierte en trata de personas (Castillo, 2003).

Los relatos de los migrantes entrevistados denotan que han vivido los dos tipos de riesgos descritos en el primer párrafo: circunstanciales y arbitrarios. Los primeros en el transporte utilizado y por las enfermedades causadas por las inclemencias del clima. Los segundos en la extorsión por policías y agentes de migración, las agresiones de grupos criminales (pandilleros, mafias y narcotraficantes y de ciudadanos mexicanos).

\section{Conceptualización de la vulnerabilidad y de las condiciones migratorias}

El concepto de vulnerabilidad se ha utilizado con fuerza en los últimos cuarenta años en las ciencias sociales, específicamente en el campo de los estudios sobre el desarrollo. Según Pérez de Armiño (2009) dicho concepto constituye un "fértil instrumento de estudio de la realidad social, de disección de sus causas profundas, de análisis multidimensional que atiende no sólo a lo económico, sino también a los vínculos sociales, el peso político, el entorno físico y medioambiental o las relaciones de género, entre otros factores" (p. 2). De ahí que sea un punto de partida importante para orientar las políticas públicas en cuestiones de desarrollo, así como las intervenciones de acción humanitaria.

Pérez de Armiño (2009) define la vulnerabilidad como:

El nivel de riesgo que afronta una familia o individuo a perder la vida, sus bienes y propiedades o su sistema de sustento, ante una posible catástrofe. Dicho nivel guarda también correspondencia con el grado de dificultad para recuperarse después de tal catástrofe, la indefensión o falta de medios para afrontar la situación sin pérdidas perjudiciales (p. 11).

De esta definición se desprenden dos componentes básicos: uno externo, constituido por los riesgos o conjunto de amenazas potenciales, y otro interno, constituido por las capacidades de un individuo o grupo para afrontarlos.

En una definición simplificada, podemos decir que la vulnerabilidad es el nivel de exposición a riesgos y la dificultad para afrontarlos. ${ }^{3}$ Los riesgos están asociados

Un análisis más complejo de la vulnerabilidad incluye el conjunto de factores estructurales que condicionan y constriñen las capacidades del individuo y su posibilidad de respuesta, esto es, las causas profundas de la vulnerabilidad (Vogt, 2013; Pérez de Armiño, 2009). Para Pérez de Armiño la vulnerabilidad "responde a una combinación de múltiples factores geográficos, económicos, sociales, políticos 
a situaciones de inseguridad, incertidumbre y desprotección, las cuales constituyen un conjunto de amenazas potenciales o reales. La capacidad de defensa/respuesta o situación de indefensión propia de la vulnerabilidad se deriva de la carencia de recursos del individuo dadas sus características personales (edad, sexo, escolaridad, condición étnica, situación migratoria o condición socioeconómica) y de otros factores estructurales. Todo ello condiciona las posibilidades de acción y estrategia del individuo o grupo. Según Kazman (citado en Ibarra Mateos, 2009, p. 206), la capacidad de respuesta depende de los activos disponibles (conjunto de recursos materiales e inmateriales sobre los que se posee control) y de los mecanismos de apoyo externo a los que se tiene acceso. Parte de estos activos la constituyen las habilidades adaptativas o estrategias de afrontamiento que implementan los actores.

El concepto de vulnerabilidad que aquí presentamos incorpora la noción de agencia, la idea del actor social, en donde se atribuye el migrante, más allá de ser una víctima o sujeto pasivo constreñido por las condiciones estructurales adversas, "la capacidad para procesar la experiencia social y diseñar maneras de lidiar con la vida, aún bajo las formas más extremas de coerción (Long, 2007, p. 48, citado en Rivas, 2011, p. 17).

La vulnerabilidad como dificultad para resistir riesgos, o la capacidad/incapacidad para reponerse después de que estos se hayan materializado, depende de diversos factores como edad, estado de salud, escolaridad, situación socioeconómica o el capital social que posee el sujeto. Depende del conjunto de sus características personales, los activos que posea, los apoyos que recibe y las estrategias que es capaz de implementar.

\section{Capacidades $=$ características personales + activos (capital social + apoyos + habilidades adaptativas)}

Desde la perspectiva del actor social, resulta especialmente interesante el concepto de capital social como activo fundamental para enfrentar situaciones de vulnerabilidad. Este concepto, importante dentro de la teoría de redes, remite al poder de agencia de los migrantes y sus comunidades a la hora de organizar los procesos de migración e incorporación en lugares de destino. El capital social, como lazos familiares, amistosos y de la comunidad, da al migrante un gran apoyo en el proce-

y personales". Sus causas son de tres tipos: causas raíces o estructurales, los procesos de crisis a medio o corto plazo y los determinantes personales, constituyéndose de esta forma "toda una cadena explicativa que va de lo macro" y estructural a lo "micro; desde las relaciones sociales globales hasta las condiciones específicas de cada individuo" (2009, p.13). Si bien la definición de vulnerabilidad propuesta en este artículo está, por cuestiones operativas, centrada básicamente en el nivel individual, no ignora la importancia que reviste el contemplar los constreñimientos estructurales y los procesos de crisis en los lugares de origen y tránsito de los grupos migratorios estudiados. 
so migratorio; incrementa sus posibilidades migratorias y disminuye los costos o riesgos del desplazamiento. Estas redes, que constituyen el capital social, son mecanismos de transmisión de capital cultural, y proveen información importante sobre las oportunidades migratorias, las rutas y las posibles estrategias (Castles, 2010).

\section{La vulnerabilidad y sus categorías}

Con ánimo de obtener indicadores que nos ayudaran a dimensionar la vulnerabilidad en sus distintos aspectos y de manera más descriptiva que los ya mencionados por González (2012), construimos la matriz que aparece en el cuadro 3.

CUADRO 3. Perfil de vulnerabilidad = riesgos + capacidades

\begin{tabular}{lllll}
\hline & Riesgos durante la ruta & \multicolumn{2}{c}{ Capacidades } \\
\hline Naturales & $\begin{array}{l}\text { Inseguridad } \\
\text { pública }\end{array}$ & Institucionales & Condiciones personales & Activos \\
& Hechos delictivos & Control & Características físicas & Capital social \\
Climáticos & cometidos por & migratorio & y de salud & (familia, redes \\
Propios & distintos grupos & (retenes, & Perfil socioeconómico: & de apoyo, redes \\
del medio & de actores. & operativos, & - Nacionalidad & sociales, acceso \\
de transporte & estaciones & - Sexo & a información) \\
(tren, autobús, & & migratorias, etc.) & - Edad & Habilidades \\
etc.) & & & - Escolaridad & adaptativas o \\
& & & - Oficio & estrategias \\
& & & Estatus jurídico: & Apoyos \\
& & & - Identificación personal & externos \\
& & & - Documentación & \\
& & & migratoria & \\
\end{tabular}

Fuente: Elaboración propia a partir del cuaderno de INEDIM (2011).

Los riesgos responden a tres tipos de categorías: naturales (condiciones climáticas y riesgos inherentes al tipo de transporte), de inseguridad pública (incluyen la tipología de hechos delictivos a los que están expuestos u otros tipos de violencia social que los acechan) e institucionales (los obstáculos y violaciones a los derechos humanos que derivan del control migratorio). Asimismo, dividimos las capacidades en las condiciones personales del migrante (perfil socioeconómico y estatus migratorio) y sus activos disponibles (básicamente el capital social, sus habilidades adaptativas o estrategias y los apoyos externos que encuentra por la ruta).

A partir de estos elementos construimos una entrevista semiestructurada que aplicamos a 78 migrantes que viajaban por la ruta. El grupo de estudio, si bien no tiene un valor estadístico, nos permitió llegar a un efecto "saturación" en donde, al repetirse ciertas características y patrones, logramos establecer cuatro perfiles de 
vulnerabilidad básicos. Estos perfiles responden a ciertos indicadores relacionados con el género, la edad, el estatus jurídico, la profesión, la experiencia previa de viaje, las redes de apoyo, la planeación de viaje y el acceso a la información. El cuadro 4 (siguiente de la página) expone los indicadores a los que responde cada categoría de vulnerabilidad.

En los resultados de nuestra investigación (Aikin \& González, 2015, 2017) realizamos un análisis más amplio sobre la categorización de los grupos en tránsito a partir de sus condiciones personales y su capacidad de respuesta ante los peligros. Presentamos una descripción detallada de cada categoría de vulnerabilidad (moderada, media, alta y extrema) y ponderamos el peso específico de los factores que inciden sobre las mismas, como género, edad, capital social y nivel de profesionalización, entre otros. Asimismo, además de analizar, a partir de historias de vida, cómo el factor género configura experiencias migratorias sustancialmente diferentes, elaboramos un mapa de riesgos a lo largo de la ruta en el que incluimos descripciones de los actores que los generan.

\section{Consideraciones finales}

El migrante que transita la ruta Occidente de México huye de situaciones de pobreza y violencia en su lugar de origen y tiene poco conocimiento geográfico del país y de los riesgos con los que se puede cruzar. No obstante, opta por afrontar peligros graves y desconocidos frente a la dificultad para sobrevivir en su propio país. Estamos hablando, sin duda, de una población que viaja en condiciones precarias e intensa vulnerabilidad. No obstante, más allá de estas generalizaciones, podemos observar que existen diferencias significativas entre los migrantes y los recursos que poseen para afrontar la adversidad. En este sentido, resulta útil desmenuzar la vulnerabilidad en sus distintas categorías o niveles para comprender las características y condiciones específicas de los distintos grupos en tránsito. Esto, además de aportar un análisis más profundo acerca de uno de los fenómenos sociales más trágicos que enfrenta México, permite el diseño de políticas públicas definidas, diferenciadas y más dirigidas a las necesidades específicas de esta población.

Si bien este trabajo se basa en las entrevistas realizadas a migrantes mexicanos, guatemaltecos, hondureños y salvadoreños, este pequeño grupo de estudio no refleja la complejidad real de todos los flujos que la transitan en distintas direcciones ni cuantifica todos los peligros a los que se enfrentan. La gran distancia que deben recorrer, los riesgos registrados y los escasos apoyos de comedores o de casas de migrantes que existen en esta ruta hacen que los migrantes sean sujetos muy vulnerables. 
CUADRO 4. Niveles de vulnerabilidad e indicadores para migrantes en tránsito

\begin{tabular}{|c|c|c|c|c|}
\hline Indicadores & Moderada & Media & Alta & Extrema \\
\hline Género & $\begin{array}{l}\text { Hombres y } \\
\text { mujeres }\end{array}$ & Hombres & $\begin{array}{l}\text { Hombres y } \\
\text { mujeres }\end{array}$ & $\begin{array}{l}\text { Hombres } \\
\text { menores y } \\
\text { mujeres }\end{array}$ \\
\hline Edad & $25-40$ & $25-40$ & $18-45$ & $0-55$ \\
\hline Estatus jurídico & $\begin{array}{l}\text { Documentado en } \\
\text { su tránsito por } \\
\text { México }\end{array}$ & $\begin{array}{l}\text { Indocumentado } \\
\text { en su tránsito por } \\
\text { México }\end{array}$ & $\begin{array}{l}\text { Indocumentado } \\
\text { en su tránsito por } \\
\text { México }\end{array}$ & $\begin{array}{l}\text { Indocumentado } \\
\text { en su tránsito por } \\
\text { México }\end{array}$ \\
\hline $\begin{array}{l}\text { Profesión } \\
\text { consolidada }\end{array}$ & $\begin{array}{l}\text { No } \\
\text { necesariamente }\end{array}$ & Sí & No & No \\
\hline $\begin{array}{l}\text { Medio de } \\
\text { transporte }\end{array}$ & Autobús y van & $\begin{array}{l}\text { Mixto autobús/ } \\
\text { tren }\end{array}$ & Tren & Tren \\
\hline $\begin{array}{l}\text { Experiencia } \\
\text { previa de viaje }\end{array}$ & $\begin{array}{l}\text { No } \\
\text { necesariamente }\end{array}$ & Sí & No & No \\
\hline Redes de apoyo $^{1}$ & Densidad alta & Densidad media & Densidad baja & $\begin{array}{l}\text { Muy escasas o } \\
\text { inexistentes }\end{array}$ \\
\hline $\begin{array}{l}\text { Planeación de } \\
\text { viaje }^{2}\end{array}$ & Alta & Media & Baja & $\begin{array}{l}\text { Muy escasa o } \\
\text { inexistente }\end{array}$ \\
\hline $\begin{array}{l}\text { Acceso a la } \\
\text { información y } \\
\text { comunicación }{ }^{3}\end{array}$ & Alta & Media & Baja & $\begin{array}{l}\text { Muy escasa o } \\
\text { inexistente }\end{array}$ \\
\hline \multirow{5}{*}{$\begin{array}{l}\text { Estrategias de } \\
\text { supervivencia }\end{array}$} & Viajar en autobús & Viajar en autobús & Charolear & Charolear \\
\hline & Contratar coyote & Charolear & \multirow{4}{*}{$\begin{array}{l}\text { Viajar en grupo } \\
\text { que se forma } \\
\text { de manera } \\
\text { espontánea } \\
\text { durante el viaje }\end{array}$} & \multirow{4}{*}{$\begin{array}{l}\text { Viajar en grupo } \\
\text { que se forma } \\
\text { de manera } \\
\text { espontánea } \\
\text { durante el viaje }\end{array}$} \\
\hline & & $\begin{array}{l}\text { Trabajar durante } \\
\text { el tránsito }\end{array}$ & & \\
\hline & & $\begin{array}{l}\text { Viajar en grupo } \\
\text { desde origen }\end{array}$ & & \\
\hline & & $\begin{array}{l}\text { Rodear zonas } \\
\text { peligrosas }\end{array}$ & & \\
\hline
\end{tabular}

Fuente: Elaboración propia a partir del trabajo de campo de esta investigación.

$1 \quad$ Alta: redes de tres tipos: origen, destino y tránsito. Media: redes de dos tipos: en lugar de destino y origen, a veces tránsito. Baja: solo un tipo de red, a menudo solo en origen y sin redes en destino. Cuenta con redes espontáneas creadas durante el tránsito. Muy escasas o inexistentes: migrante no tiene redes o solo cuenta con las espontáneas creadas durante el tránsito.

2 Alta: migrante manifiesta haber planeado ahorrando dinero, contratando coyote, consultando mapas y a otros migrantes, tiene claro el destino en Estados Unidos, dónde trabajará y cuánto tiempo se quedará en ese destino. Media: planeó viaje, ahorró, a veces consultó mapas, pero no contrató coyote. Tiene claro el lugar de destino, pero no necesariamente en dónde trabajará o cuánto tiempo se quedará en destino. Baja y muy escasa o inexistente: planeación muy precaria, a veces ahorro para el viaje y consulta a otros migrantes en lugar de origen. Otras veces viaja sin información previa (salvo la de boca a boca), sin uso de coyote y sin tener claro lugar de destino o tipo de trabajo que realizará.

3 Se gradúa dependiendo de a cuántas fuentes accedió antes y durante el viaje; uso de celular, whatsapp, correo electrónico, mapas, noticias, información proveniente de otros migrantes en origen o tránsito. Alta: acceso a cuatro o más fuentes. Media: acceso a máximo tres fuentes. Baja: acceso máximo a dos fuentes. Muy escasa: acceso a una fuente, generalmente información de otros migrantes. 
En el grupo de estudio pudimos encontrar, además de la vulnerabilidad general propia de cualquier migrante pobre en tránsito que utiliza el tren como medio de transporte, la particular de personas que viajan de manera irregular por un país extraño, la de menores de edad que viajan solos o la de mujeres que igualmente viajan solas y cuyo nivel de riesgo es superior solo por el hecho de ser mujeres. No obstante, estos grupos migratorios poseen ciertos recursos (materiales e inmateriales) y logran generar estrategias de supervivencia ante la adversidad. Así, desde la óptica del actor social, se recupera la capacidad de agencia de estos individuos y logramos ir más allá de la imagen de victimización que suelen subrayar los medios, reportes y análisis académicos que retratan este fenómeno.

Para terminar, es importante decir que las vulnerabilidades encontradas habían sido ya documentadas por otros informes y reportes (CNDH, 2009; Amnistía Internacional, 2010; FM4 Paso Libre, 2013). Sin embargo, ese trabajo siempre se ha hecho de manera generalizada a toda la migración de tránsito y de todas las rutas. La pertinencia y particularidad de los resultados de esta investigación ha sido la categorización de la vulnerabilidad por niveles (moderada, media, alta y extrema) con el análisis propio de las capacidades y estrategias de las personas migrantes; lo que nos deja ver con rostros más concretos quiénes son los que pasan por México y, específicamente, por la ruta Occidente, así como lo que significa migrar por estos caminos.

\section{Agradecimientos}

Agradecemos y valoramos la colaboración de varias personas e instituciones que han facilitado enormemente esta investigación: los estudiantes del Instituto Tecnológico y de Estudios Superiores de Occidente (ITESo) Alejandra Sandoval, Óscar Limón, Gabriela Rangel; a las voluntarias de Iniciativa Kino Ashley Edgette y Natalia; al Programa de Asuntos Migratorios de ITESo (PRAMI); a FM4 Paso Libre; al proyecto Iniciativa Kino para la Frontera, y a Daniel David González Arias, quien elaboró los mapas.

\section{Referencias}

Aikin, O. \& González, A. (2015). Migración de tránsito por la ruta del Occidente de México: actores, riesgos y perfiles de vulnerabilidad. Migración y Desarrollo, 13(24), 81-115.

Aikin, O. \& González, A. (2017). Tránsito migratorio por el occidente de México: la ruta, sus migrantes y los riesgos. En Aikin, O. \& González, A. (coords.), Procesos migratorios en el occidente de México. Guadalajara: ITEso. 
Amnistía Internacional (2010). Víctimas invisibles. Migrantes en movimiento en México. Londres: Amnistía Internacional.

Casillas, R. (2008). Las rutas de los centroamericanos por México, un ejercicio de caracterización, actores principales y complejidades. Migración y Desarrollo, 10, 157-174.

Castillo, M. A. (2003). Migraciones en el hemisferio. Consecuencias y relación con las políticas sociales. Santiago de Chile: CEPAL.

Castles, S. (2010). Understanding global migration: a social transformation perpective. Journal of Ethnic and Migration Studies, 36(10), 1565-1586.

Comisión Nacional de los Derechos Humanos (CNDH) (2009). Informe especial de la Comisión Nacional de los Derechos Humanos sobre los casos de secuestro en contra de migrantes. México: CNDH.

Encuesta sobre Migración en la Frontera Norte de México, (EMIF Norte) (2011). México: El Colegio de la Frontera Norte, Secretaría del Trabajo y Previsión Social, Consejo Nacional de Población, Unidad de Política Migratoria, Secretaría de Relaciones Exteriores. Recuperado de www.colef.mx/emif (consultado el 12 de diciembre de 2014).

Encuesta sobre Migración en la Frontera Sur de México, (EMIF Sur) (2012). Encuesta sobre migración en la Frontera Sur de México, 2009. Serie histórica 2004-2009. México: Secretaría de Gobernación, Instituto Nacional de Migración, Consejo Nacional de Población, El Colegio de la Frontera Norte (COLEF), Secretaría de Relaciones Exteriores, Secretaría del Trabajo y Previsión Social.

FM4 Paso Libre (2013). Migración en tránsito por la zona metropolitana de Guadalajara: actores, retos y perspectivas desde la experiencia de FM4 Paso Libre. Guadalajara: Prometeo Editores.

Foote, J. y Small, M. (2013). Inseguridad permanente: abusos contra centroamericanos en México. Washington, DC: Jesuit Refugee Service USA. Recuperado de https://www. jrsusa.org/assets/Publications/File/Inseguridad_Permanente_web.pdf_(consultado el 20 de junio de 2014).

González, A. (2012). Migración de tránsito por México: una cartografía del movimiento territorial. En Lizardi, A. \& Ortiz, K. (coords.), Cartografías del movimiento. Bosquejos de espacios creados por recorridos de individuos y artefactos. México: Universidad de Guadalajara.

Ibarra Mateos, L. (2009). Diez años pasaron ya y sigo siendo un ilegal: la migración internacional como expresión de vulnerabilidad social. En Meza González, L. \& Cuéllar Álvarez, M. (comps.), La vulnerabilidad de los grupos migrantes en México. Ciudad de México: Universidad Iberoamericana.

Instituto de Estudios y Divulgación sobre Migración (INEDIM) (2011). Seguridad para el migrante: una agenda por construir. Ciudad de México: INEDIM.

Long, N. (2007). Sociología del desarrollo: una perspectiva centrada en el actor. Ciudad de México: COLSAN/CIESAS. 
Machuca, R., director de programas en México para la Iniciativa Kino (2014, 28 de febrero). Entrevista. Nogales, Sonora.

Oficina de las Naciones Unidas contra la Droga y el Delito (UnDoc) (2012). Delincuencia organizada transnacional en Centroamérica y el Caribe. Una evaluación de las amenazas. Viena: Oficina de las Naciones Unidas contra la Droga y el Delito.

Pérez de Armiño, K. (2009). Vulnerabilidad y desastres. Causas estructurales y procesos de la crisis en África. Cuadernos de Trabajo de Hegoa, 24, 1-64. Recuperado de http:// publ.hegoa.efaber.net/assets/pdfs/123/Cuaderno_de_trabajo_24.pdf?130400206 (consultado el 30 de noviembre de 2013).

Rivas, J. (2011). ¿Víctimas nada más? Migrantes centroamericanos en el Soconusco, Chiapas. Nueva Antropología, 24 (74), 9-38.

Vogt, W. (2013). Crossing Mexico: structural violence and the commodification of undocumented Central American migrants. American Ethnologist, 40(4), 764-780). Recuperado de http://www.academia.edu/5073265/Crossing_Mexico_Structural_violence_and_the_commodification_of_undocumented_Central_American_migrants (consultado el 20 de abril de 2014). 